\title{
Analytic approximations to Kelvin functions with applications to electromagnetics
}

\author{
L. Brualla* \\ Departament de Física i Enginyeria Nuclear, Universitat Politècnica de Catalunya, \\ Sor Eulàlia d'Anzizu s/n, E-08034 Barcelona, Spain. \\ P. Martin \\ Departamento de Física, Universidad Simón Bolivar, Apartado 89000, Caracas 1080A, Venezuela.
}

\begin{abstract}
We present analytical approximations for the real Kelvin function ber $x$ and the imaginary Kelvin function bei $x$, using the two-point quasifractional approximation procedure. We have applied these approximations to the calculation of the current distribution within a cylindrical conductor. Our approximations are simple and accurate. The infinite number of roots is also obtained with the approximation and the precision increases with the value of the root. Our results could find useful applications in problems where analytical approximations of the Kelvin functions are needed.
\end{abstract}

\section{INTRODUCTION}

Within the scope of special functions, Kelvin functions appear early in the literature [1] [2]. They are derived from the Bessel functions [3] of complex argument and many formulas can be found. In addition to their importance in Mathematics they have, also, a broad

\footnotetext{
${ }^{*}$ Llorenc.Brualla@upc.es
} 
spectrum of application in Physics. For instance, in the analysis of the current distribution in cylindrical conductors due to wave propagation [4]. A more interesting application is the problem of determining the equivalent impedance of a cylindrical conductor which is given in terms of ber $x$ and bei $x$. Kelvin functions also arose in the problem of the waveimpedance of cylindrically layered conductors for application in nondestructive testing [5]. The temperature distribution in cylindrical conductors due to an alternative current also involves ber $x$ and bei $x$ [6]. Kelvin functions also appear in other fields (i.e., fluid mechanics) but in this paper we will concentrate on the first electromagnetic application mentioned above.

One of the problems we have found in the applications of Kelvin functions is that their behaviour is rather complicated. Kelvin functions are oscillatory and the amplitude of the oscillations increases rapidly. In spite of the already existing tables, still the functions are part of integrands where the tables are difficult to use. The programs to compute these functions are also too elaborated.

It seems convenient to have a good approximation to the functions, but the conventional approximation methods do not give good results because of the pathological behaviour of the functions. However, in this paper it is shown how those difficulties can be surmounted by using the recently published method of two-point quasifractional approximations [8] [9]. This has allowed us to find analytic approximations to Kelvin functions with good accuracy, and furthermore, the same analytic approximation is valid for the whole range of positive values of the variable.

Power series and asymptotic expansions are required in order to obtain these approximations, thus we start analysing those expansions to proceed further away. This will be done in Sec. 2, where the form of the approximation will be also derived. Later, the material of this paper will be arranged as follows: the calculation of the parameters for the two-point quasifractional approximation of ber $x$ is carried out in Sec. 3. Sec. 4 devotes its attention to the analysis of the results of the approximation. The same procedure applied to ber $x$ in previous sections is employed in the calculation of bei $x$. That calculation and the discussion 
of the results obtained for bei $x$ are performed in Sec. 5. In Sec. 6 we show an immediate application to engineering. Finally, Sec. 7 aims its attention to discussion and conclusions of the paper.

\section{TWO-POINT QUASIFRACTIONAL APPROXIMATION FORM FOR THE REAL KELVIN FUNCTION}

As it is well known, the real Kelvin function is defined as [2]

$$
\text { ber } x=\Re\left(J_{0}(i \sqrt{i} x)\right)
$$

where $J_{0}(z)$ is the zeroth order Bessel function.

The power series is given by

$$
\text { ber } x=1-\frac{\left(\frac{1}{4} x^{2}\right)^{2}}{(2 !)^{2}}+\frac{\left(\frac{1}{4} x^{2}\right)^{4}}{(4 !)^{2}}-\cdots
$$

The asymptotic expansion of ber $x$ has been also given in several references. Our interest here is mainly in the leading term, which can be written as

$$
\text { ber } x \sim \frac{e^{x / \sqrt{2}}\left(\frac{\sqrt{2+\sqrt{2}}}{2} \cos \left(\frac{x}{\sqrt{2}}\right)+\frac{\sqrt{2-\sqrt{2}}}{2} \sin \left(\frac{x}{\sqrt{2}}\right)\right)}{\sqrt{2 \pi x}} \text {. }
$$

Once the potential series (2) and the asymptotic expansion (3) have been obtained, it is possible to determine the form of the quasifractional approximations of the function ber $x$, which has an essential singularity at infinity. That kind of singularity is characteristic of all the hypergeometric confluent functions. The asymptotic expansion (3) picks up this singularity and shows it up through a branch point at infinity together with essential singularities at infinity of the exponential functions. Since the branch points come up in pairs, the leading term of the asymptotic expansion shows also a second branch point at $x=0$. However, the behaviour of the function ber $x$ is regular at $x=0$, therefore the asymptotic form in (3) is not suitable as an approximation for ber $x$ in the region near zero. In order to pursue the goal of picking up the right behaviour at infinity, but not to introduce undesired 
singularities in the zone of interest, a suitable auxiliary function has to be chosen. The auxiliary function should have the right ramification form at infinity, and the second branch point located outside the zone of interest (i.e., the negative axis)

Only powers of the form $4 n$ appear in the potential series of ber $x$. In order to achieve the adequate efficency in the approximation, we should find such auxiliary functions and fractional approximations whose potential series only have exponents multiple of 4 .

Since the second branch point introduced with the asymptotic expansion must be outside the zone of interest, it is possible to choose as an auxiliary function $A_{1}(x)=1 / \sqrt{1+x}$; however, that auxiliary function would not be efficient, since undesired power terms as $x^{2}, x^{3}, \ldots$ would appear. A more suitable auxiliary function would be $\tilde{A}_{1}(x)=1 / \sqrt[8]{1+x^{4}}$ or $\tilde{A}_{2}(x)=1 / \sqrt[8]{1+\tau^{4} x^{4}}$. However, in the last case the parameter $\tau$ could be chosen in a convenient way in order to get better accuracy with the approximation, as we will discuss later, this can be considered as a free parameter.

The other singularity that must be introduced through the auxiliary functions is of the form $e^{x / \sqrt{2}} \cos \left(\frac{x}{\sqrt{2}}\right)$ and $e^{x / \sqrt{2}} \sin \left(\frac{x}{\sqrt{2}}\right)$. The efficiency criteria limit the auxiliary functions by forcing them to have exponents of the form $4 n$. The above stated line of thought leads us to choose as convenient auxiliary functions

$$
\cosh \left(\frac{x}{\sqrt{2}}\right) \cos \left(\frac{x}{\sqrt{2}}\right)
$$

and

$$
\frac{\sinh \left(\frac{x}{\sqrt{2}}\right) \sin \left(\frac{x}{\sqrt{2}}\right)}{x^{2}}
$$

Once the auxiliary functions are chosen as above, the fractional approximations automatically have only powers multiple of 4 .

All the previous considerations lead to the following form of the two-point quasifractional approximation for the real Kelvin function

$$
\text { ber } x=\frac{\sum_{k=0}^{n} p_{k} x^{4 k} \cosh \left(\frac{x}{\sqrt{2}}\right) \cos \left(\frac{x}{\sqrt{2}}\right)+\frac{\sqrt{1+\alpha^{2} x^{4}}}{x^{2}} \sum_{k=0}^{n} P_{k} x^{4 k} \sinh \left(\frac{x}{\sqrt{2}}\right) \sin \left(\frac{x}{\sqrt{2}}\right)}{\left(1+\sum_{k=1}^{n} q_{k} x^{4 k}\right) \sqrt[8]{1+\tau^{4} x^{4}}} .
$$


The auxiliary function $\sqrt{1+\alpha^{2} x^{4}}$ has to be introduced in order to cancel the $1 / x^{2}$ behaviour at infinity. With this function we also define a second free parameter $\alpha$.

\section{CALCULATION OF THE TWO-POINT QUASIFRACTIONAL APPROXIMATION TO THE REAL KELVIN FUNCTION}

Here we will consider only the simplest approximation to ber $x$, thus $n$ will be one and the approximation in eq. (6) will be reduced to

$$
\text { ber } x=\frac{\left(p_{0}+p_{1} x^{4}\right) \cosh \left(\frac{x}{\sqrt{2}}\right) \cos \left(\frac{x}{\sqrt{2}}\right)+\frac{\sqrt{1+\alpha^{2} x^{4}}}{x^{2}}\left(P_{0}+P_{1} x^{4}\right) \sinh \left(\frac{x}{\sqrt{2}}\right) \sin \left(\frac{x}{\sqrt{2}}\right)}{\left(1+q x^{4}\right) \sqrt[8]{1+\tau^{4} x^{4}}} .
$$

The ideas developed on the latest papers on quasifractional approximations are in the sense of not to determine all the coefficients through the powers series and the asymptotic expansions but to leave one or two free parameters - $\alpha$ and $\tau$ - that can be determined by minimising the maximum absolute error. It is clear that other methods to measure the discrepancy between the approximant and the exact function can also be used, such as the Lebesgue's integral of the quadratic difference, or other more elaborated methods using Lebesgue's integrals of the $p$-powers of the difference. However, for us, the most convenient way to measure that discrepancy has been the maximum absolute error.

The main reason for using free parameters is to avoid the characteristic defects of classic Padé [7], also common in quasifractional approximations. Defects are when an extraneous pole appears near a zero in the numerator. That is, there is a zero in the numerator in $x_{0}$ and another zero in the denominator in $x_{1}$ and the difference $\left|x_{0}-x_{1}\right|$ is very small. Due to that problem, the approximation is in good agreement with the function in the whole zone of interest, except when close to $x_{0}$ and $x_{1}$, where it goes to $+\infty,-\infty$ and 0 .

In order to determine the five unknowns $p_{0}, P_{0}, p_{1}, P_{1}, q$ three terms of the power series (2) and two terms of the asymptotic expansion (3) will be used. So a linear system of five equations with five unknowns is obtained. The solutions will be given in terms of $\alpha$ and $\tau$.

Looking at the exponential term in (3), the variable $x / \sqrt{2}$ appears as more convenient. 
Thus the power series (2) will be written now as

$$
\operatorname{ber}(x \sqrt{2})=1-\frac{1}{16} x^{4}+\frac{1}{9216} x^{8}-\cdots
$$

since we are using only three terms of this series we do not need to go further away than $x^{8}$.

Now, to compare the power series of ber $x$ and bẽr $x$, we will first multiply both functions by $\left(1+q x^{4}\right)$ in order to rationalise the right hand side. Furthermore the auxiliary functions appearing in (7) and defined in (44) and (5), should be replaced by their respective power series. Thus we obtain

$$
\begin{array}{r}
\left(1+q x^{4}\right)\left(1-\frac{1}{16} x^{4}+\frac{1}{9216} x^{8}\right)= \\
\left(1-\frac{1}{8} \tau^{4} x^{4}+\frac{9}{128} \tau^{8} x^{8}\right)\left(\left(1-\frac{1}{24} x^{4}+\frac{1}{40320} x^{8}\right)\left(p_{0}+p_{1} x^{4}\right)+\right. \\
\left.\left(\frac{1}{2}-\frac{1}{720} x^{4}+\frac{1}{3628800} x^{8}\right)\left(1+\frac{1}{2} \alpha^{2} x^{4}-\frac{1}{8} \alpha^{4} x^{8}\right)\left(P_{0}+P_{1} x^{4}\right)\right)+O\left(x^{12}\right)
\end{array}
$$

where the first term in the first parenthesis on the right hand side is the power expansion of $\sqrt[8]{1+\tau^{4} x^{4}}$, and constant factors have been absorbed in the definition of the parameters $\tau$ and $q$.

Equalising now the powers $x^{0}, x^{4}$ and $x^{8}$ in both sides of the equation we obtain

$$
\begin{gathered}
p_{0}+\frac{1}{2} P_{0}=1 \\
\left(-\frac{1}{24}-\frac{1}{8} \tau^{4}\right) p_{0}+\left(-\frac{1}{16} \tau^{4}-\frac{1}{720}+\frac{1}{4} \alpha^{2}\right) P_{0}+\frac{1}{2} P_{1}+p_{1}-q=-\frac{1}{16} \\
\left(\frac{1}{192} \tau^{4}+\frac{1}{40320}+\frac{9}{128} \tau^{8}\right) p_{0}+ \\
\left(\frac{1}{5760} \tau^{4}+\frac{9}{256} \tau^{8}-\frac{1}{16} \alpha^{4}-\frac{1}{32} \tau^{4} \alpha^{2}-\frac{1}{1440} \alpha^{2}+\frac{1}{3628800}\right) P_{0}+ \\
\left(-\frac{1}{24}-\frac{1}{8} \tau^{4}\right) p_{1}+\left(-\frac{1}{16} \tau^{4}-\frac{1}{720}+\frac{1}{4} \alpha^{2}\right) P_{1}+\frac{1}{16} q=\frac{1}{9216}
\end{gathered}
$$

The leading term of the asymptotic expansion of berr $x$ is

$$
\text { ber } x \sim e^{x / \sqrt{2}}\left[\frac{1}{2} \frac{p_{1} \cos \left(\frac{x}{\sqrt{2}}\right)+P_{1} \alpha \sin \left(\frac{x}{\sqrt{2}}\right)}{q \sqrt{\tau x}}\right] \text {. }
$$

And comparing now the leading terms of ber $x$, we obtain 


$$
\begin{aligned}
& p_{1}=\frac{\sqrt{2+\sqrt{2}} \sqrt{\tau} q}{\sqrt{2 \pi}} \\
& P_{1}=\frac{\sqrt{2-\sqrt{2}} \sqrt{\tau} q}{\sqrt{2 \pi} \alpha}
\end{aligned}
$$

Equations (10), (11), (12), (14) and (15) determine the values of $p_{0}, P_{0}, p_{1}, P_{1}$ and $q$ as functions of $\alpha$ and $\tau$.

When these equations are solved, an expression for each parameter is obtained in terms of $\alpha$ and $\tau$. And an approximation $\tilde{\operatorname{ber}}(x, \alpha, \tau)$ is defined for each value of $\alpha$ and $\tau$.

Given the initial values $\alpha_{0}$ and $\tau_{0}$, we numerically determine the value of the function $|\tilde{\operatorname{ber}}(x, \alpha, \tau)-\operatorname{ber}(x)|$ and we select the maximum value of this function, which will be the maximum absolute error. Therefore, the maximum absolute error, $\epsilon(\alpha, \tau)$, will be a function of $\alpha$ and $\tau$. Looking now at $\epsilon(\alpha, \tau)$ as a function, we can determine the values $\alpha_{m}$ and $\tau_{m}$ which minimise this function as a two variable function. That procedure has been followed and the values $\alpha_{m}$ and $\tau_{m}$ are found as

$$
\begin{aligned}
\alpha_{m} & =0.98 \\
\tau_{m} & =0.8367
\end{aligned}
$$

In order to avoid the defect in the approximation we have to consider only positive values of $q$, thus there is no problem with positive values for the variable $x$ which is the region of interest. This must be taken into account when sweeping through $\alpha$ and $\tau$ in the minimising procedure. Local minima which yield negative values for $q$ must be discarded.

Using these values of $\alpha$ and $\tau$ in (10), (11), (12), (14) and (15), the parameters $p_{0}, P_{0}, p_{1}, P_{1}$ and $q$ are determined and the results are

$$
\begin{gathered}
q=27627.311660 \\
p_{0}=-9750.649914 \\
P_{0}=19503.300340 \\
p_{1}=18628.544300 \\
P_{1}=7873.669071
\end{gathered}
$$




\section{ACCURACY OF THE TWO-POINT QUASIFRACTIONAL APPROXIMATION OF THE REAL KELVIN FUNCTION}

As it has been stated before, the discrepancy of the approximation will be determined by $\Delta$ ber $x=\operatorname{ber} x-\operatorname{ber} x$.

A plot of the real Kelvin function, together with the approximation and ten times the absolute error is displayed in Figure 1.

Besides, a table of the first five roots of the Kelvin function and the approximation is introduced.

Notice that the highest relative error in the roots is the first one. Quasifractional ap-

proximations to any function have normally their worst accuracy for values of order one. The behaviour of the approximation becomes more accurate as $x$ increases.

Another two measures of the good agreement of the approximation with the real Kelvin function is the relative difference of the position in $x$ of the maxima of the function and the relative error of the amplitude of each maximum. The following table shows the position in $x$ of the first five maxima of ber $x$ and $\tilde{\operatorname{ber}} x$ as $\operatorname{ber}^{\prime} x$ and $\tilde{b e r}^{\prime} x$ respectively, and the relative difference between them. The value of each of the first five maxima is also shown, appearing as $\operatorname{ber}_{m} x$ and $\tilde{\operatorname{ber}}_{m} x$ for the real Kelvin function and the approximation respectively. The relative error of those figures also appears.

Notice that all the relative errors, no matter what is being measured (i.e., the roots, the position of the maxima, the amplitude) decrease as $x$ becomes greater.

\section{CALCULATION OF THE TWO-POINT QUASIFRACTIONAL APPROXIMATION FOR THE IMAGINARY KELVIN FUNCTION}

The same line of thought carried out for the real Kelvin function will be followed in this section, since the form of the asymptotic expansions and the power series for both ber $x$ and bei $x$ are very similar. 
The imaginary Kelvin function is defined as [2]

$$
\text { ber } x=\Im\left(J_{0}(i \sqrt{i} x)\right)
$$

where $J_{0}(z)$ is the zeroth order Bessel function. The power series of bei $x$ is given by

$$
\text { bei } x=\frac{1}{4} x^{2}-\frac{\left(\frac{1}{4} x^{2}\right)^{3}}{(3 !)^{2}}+\frac{\left(\frac{1}{4} x^{2}\right)^{5}}{(5 !)^{2}}-\cdots
$$

The asymptotic expansion of bei $x$ has been also given in several references. Again, our interest here lays mainly on the leading term, which can be written as

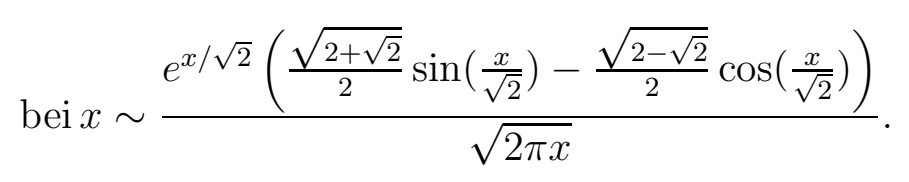

It is a characteristic of hypergeometric confluent functions to have an essential singularity at infinity. The asymptotic expansion picks that singularity up and shows it through a branch point at infinity with the exponential functions, in the same way that happened for ber $x$.

The power series of bei $x$ goes as $x^{2}, x^{6}, x^{10}, \ldots$ In order to have an efficient approximation, the auxiliary functions chosen for moving the artificially introduced branch point out of the positive axis and for introducing the singularities that appear in the asymptotic expansion should have a power series of the same form as bei $x$.

Following the same stream of thought used in determining the form of ber $x$, a correct form for the quasifractional approximation of bei $x$ is

$$
\tilde{\text { bei } x}=\frac{\frac{x^{2}}{\sqrt{1+\bar{\alpha}^{2} x^{4}}} \sum_{k=0}^{n} \bar{p}_{k} x^{4 k} \cosh \left(\frac{x}{\sqrt{2}}\right) \cos \left(\frac{x}{\sqrt{2}}\right)+\sum_{k=0}^{n} \bar{P}_{k} x^{4 k} \sinh \left(\frac{x}{\sqrt{2}}\right) \sin \left(\frac{x}{\sqrt{2}}\right)}{\left(1+\sum_{k=1}^{n} \bar{q}_{k} x^{4 k}\right) \sqrt[8]{1+\bar{\tau}^{4} x^{4}}}
$$

where $\bar{\alpha}$ and $\bar{\tau}$ are free parameters, introduced for the same reason explained for ber $x$. We are going to use only the simplest form of the approximation, thus $n=1$ will be substituted in the previous equation. Thus the two-point quasifractional approximation of bei $x$ will give

$$
\text { bei } x=\frac{\frac{x^{2}}{\sqrt{1+\bar{\alpha}^{2} x^{4}}}\left(\bar{p}_{0}+\bar{p}_{1} x^{4}\right) \cosh \left(\frac{x}{\sqrt{2}}\right) \cos \left(\frac{x}{\sqrt{2}}\right)+\left(\bar{P}_{0}+\bar{P}_{1} x^{4}\right) \sinh \left(\frac{x}{\sqrt{2}}\right) \sin \left(\frac{x}{\sqrt{2}}\right)}{\left(1+\bar{q} x^{4}\right) \sqrt[8]{1+\bar{\tau}^{4} x^{4}}} .
$$


From now on, exactly the same procedure has been followed in determining the set of five equations used for finding the values of the parameters $\bar{p}_{0}, \bar{P}_{0}, \bar{p}_{1}, \bar{P}_{1}, \bar{q}$ in terms of $\bar{\alpha}$ and $\bar{\tau}$, and for minimising the discrepancy of the approximation.

When dealing with quasifractional approximations it is common to take as an initial ansatz $\alpha$ and $\tau$ equal to 1 . This choice proved to be a valid starting point for berr $x$, even though smaller differences are obtained when using the minimised $\overline{\alpha_{m}}$ and $\overline{\tau_{m}}$ given in the previous section. The bei $x$ function is somewhat more pathological since the choice $\bar{\alpha}=\bar{\tau}=1$ yields a negative value of $\bar{q}$, making the initial ansatz useless.

For minimising the maximum absolute error of the two-point quasifractional approximation of bei $x$ a downhill simplex method has been used [10]. The values obtained for the parameters are

$$
\begin{aligned}
\overline{\alpha_{m}} & =3.00 \\
\overline{\tau_{m}} & =3.00 \\
\bar{q} & =19.11054940 \\
\bar{p}_{0} & =-7.21235948 \\
\bar{P}_{0} & =15.42471896 \\
\bar{p}_{1} & =-30.32038957 \\
\bar{P}_{1} & =24.39996523
\end{aligned}
$$

The plots of bei $x$, bei $x$ and ten times the absolute error $(10 \Delta \tilde{\text { bei } x}=10(\tilde{\text { bei }} x-$ bei $x)$ ) are shown in Figure 2.

The relative error of the first root is smaller than the one of the first root of ber $x$ because the value of the variable $x$ is greater (i.e., the first root of bei $x$ it is not as close to zero as the one of ber $x$ ). The accuracy for all the roots of ber $x$ and bei $x$ is very high in any case.

Once again, the relative error related to the position of the first five maxima of the function and the error of their amplitude will be calculated in the same way performed for ber $x$. The notation is exactly the same followed in the calculation of error for ber $x$ before 
in Sec. 4, but adapted for the function bei $x$.

\section{ANALYTIC APPROXIMATION TO THE CURRENT DISTRIBUTION WITHIN A CYLINDRICAL CONDUCTOR}

The expression of the current distribution within a cylindrical conductor due to a trav-

elling wave has been given in many references. The current distribution depends on the Kelvin real and imaginary functions and is given by [4]

$$
\left|\frac{J_{z}(r)}{J_{s}}\right|=\left(\frac{\operatorname{ber}^{2}(\sqrt{2} r / \delta)+\operatorname{bei}^{2}(\sqrt{2} r / \delta)}{\operatorname{ber}^{2}\left(\sqrt{2} r_{0} / \delta\right)+\operatorname{bei}^{2}\left(\sqrt{2} r_{0} / \delta\right)}\right)^{1 / 2}
$$

where $J_{s}$ is the current density on the surface of the conductor and $r_{0}$ is the radius on the surface. The skin depth is given by $\delta$, and it depends on the frequency of the propagating wave.

By means of substitution of berr $x$ and bei $x$ in (21) it is possible to get an analytical approximation to the current distribution within a cylindrical conductor.

The analytical approximation obtained has been used for studying the current distribution within a copper wire with $r_{0}=0.5 \mathrm{~mm}$. Four different values of the frequency have been taken. The same figures and plots could be found in Marion, for comparison purposes.

The plots obtained using our approximation and those obtained using numerical computation are coincident and no difference can be found at this scale. Therefore, we are only showing the errors in Figure 3.

\section{CONCLUSIONS}

In this paper analytic approximations have been found for the function ber $x$ and bei $x$. These have been used to calculate the current distribution within a cylindrical conductor produced by the propagation of one wave of frequency $\omega$.

In spite of the strong fluctuation characteristic of the functions ber $x$ and bei $x$, the simple approximations found here not only reproduce the function with high accuracy for 
all positive values of the variable but the infinite roots or zeroes of the function are also obtained and the relative error of each root decreases with the magnitude of the root. The largest relative error of the roots for ber $x$ is about $2 \%$ and for bei $x$ is $0.5 \%$.

The measure of the discrepancy is difficult for these functions because the function fluctuates and, furthermore, the maximum of the amplitude increases and goes to infinity with $x$. Therefore, if we consider the absolute error this will go to infinity with $x$.

On the other hand, if we study the relative error, these errors will go to infinity at the roots of the function. The best way to measure the good agreement of our approximation is just through the errors of the zeroes and of the amplitude of the oscillations. It is significant that these errors decrease with $x$ despite of the large value of the function. The maximum error of the amplitude for ber $x$ is $3 \%$, and $4 \%$ for bei $x$ in the value of the function and of $0.28 \%$ and $0.80 \%$ in the site of the maximum for ber $x$ and bei $x$ respectively.

These errors clearly show that our approximation can be used for most of the applications we know until now, for example thermo-electrical corrosion in cylindrical conductors. In general, these approximations might be used whenever Kelvin functions appear and a numerical solution is not appropriate. As an example, we have applied our method to the simplest case of the current distribution in a cable, obtaining quite accurate results. 


\section{REFERENCES}

[1] Gradshteyn I S and Ryzhik I M 1965 Table of Integrals, Series and Products (New York: Academic Press)

[2] Abramowitz M and Stegun I A 1972 Handbook of Mathematical Functions (New York: Dover)

[3] Gross F B 1995 IEEE Trans. Antennas Propagat. 43904

[4] Marion J B and Heald M A 1980 Classical Electromagnetic Radiation (Orlando: Academic Press College)

[5] Wait J R and Gardner R L 1979 IEEE Trans. Instrumentation and Measurement 28 159

[6] Rolicz P 1978 J. Appl. Phys. 494363

[7] Baker G A 1975 Essentials of Padé Approximants. (New York: Academic Press)

[8] Martin P and Baker G A 1991 J. Math. Phys. 321470

[9] Martin P, Donoso G and Zamudio-Cristi J 1980 J. Math. Phys. 21280

[10] W. H. Press et al 1986 Numerical Recipes (Cambridge University Press) 


\section{TABLES}

TABLE I. Comparison between the first five roots of ber $x$ and $\tilde{\text { ber } x}$

\begin{tabular}{lcr}
\hline \hline ber $x$ roots & ber $x$ roots & Relative error $(\%)$ \\
\hline 2.84892 & 2.78620 & 2.2 \\
7.23883 & 7.22030 & 0.26 \\
11.67396 & 11.66266 & 0.094 \\
16.11356 & 16.10548 & 0.052 \\
20.55463 & 20.54834 & 0.032 \\
\hline \hline
\end{tabular}

TABLE II. Comparison between the first five maxima of ber $x$ and $\tilde{\operatorname{ber}} x$ and their derivatives.

\begin{tabular}{lccccc}
\hline \hline $\operatorname{ber}^{\prime} x$ & $\operatorname{ber}^{\prime} x$ & err.(\%) & ber $_{m} x$ & ber $_{m} x$ & err.(\%) \\
\hline 6.03871 & 6.0215 & 0.28 & -8.86404 & -8.61484 & 2.9 \\
10.51364 & 10.5027 & 0.10 & 153.782 & 151.237 & 1.7 \\
14.96844 & 14.9605 & 0.053 & -2968.68 & -2933.93 & 1.2 \\
19.41758 & 19.4114 & 0.032 & 60161.2 & 59616.8 & 0.92 \\
23.86430 & 23.8592 & 0.021 & $-1.25374 \times 10^{6}$ & $-1.2445 \times 10^{6}$ & 0.75 \\
\hline \hline
\end{tabular}

TABLE III. Comparison between the first five roots of bei $x$ and bei $x$.

\begin{tabular}{lcr}
\hline \hline bei $x$ roots & bei $x$ roots & Relative error $(\%)$ \\
\hline 5.02622 & 4.99873 & 0.55 \\
9.45541 & 9.44110 & 0.15 \\
13.89349 & 13.88400 & 0.069 \\
18.33398 & 18.32689 & 0.037 \\
22.77544 & 22.76977 & 0.024 \\
\hline \hline
\end{tabular}


TABLE IV. Comparison between the first five maxima of bei $x$ and bei $x$ and their derivatives.

\begin{tabular}{cccccc}
\hline \hline bei $^{\prime} x$ & bei $^{\prime} x$ & err.(\%) & bei $_{m} x$ & bei $_{m} x$ & err.(\%) \\
\hline 3.77320 & 3.74307 & 0.80 & 2.34615 & 2.259 & 3.9 \\
8.28099 & 8.26718 & 0.17 & -36.1654 & -35.4089 & 2.2 \\
12.74215 & 12.7329 & 0.073 & 670.16 & 660.955 & 1.4 \\
17.19343 & 17.1865 & 0.040 & -13305.5 & -13169.6 & 1.1 \\
21.64114 & 21.6356 & 0.026 & 273888 & 271661 & 0.82 \\
\hline \hline
\end{tabular}

TABLE V. Skin depth at different frequencies.

\begin{tabular}{lcr}
\hline \hline Case & $\nu=\omega / 2 \pi$ & $\delta(\mathrm{mm})$ \\
\hline 1 & $10^{3}$ & 2.1 \\
2 & $10^{4}$ & 0.66 \\
3 & $10^{5}$ & 0.21 \\
4 & $10^{6}$ & 0.066 \\
\hline \hline
\end{tabular}




\section{FIGURES}

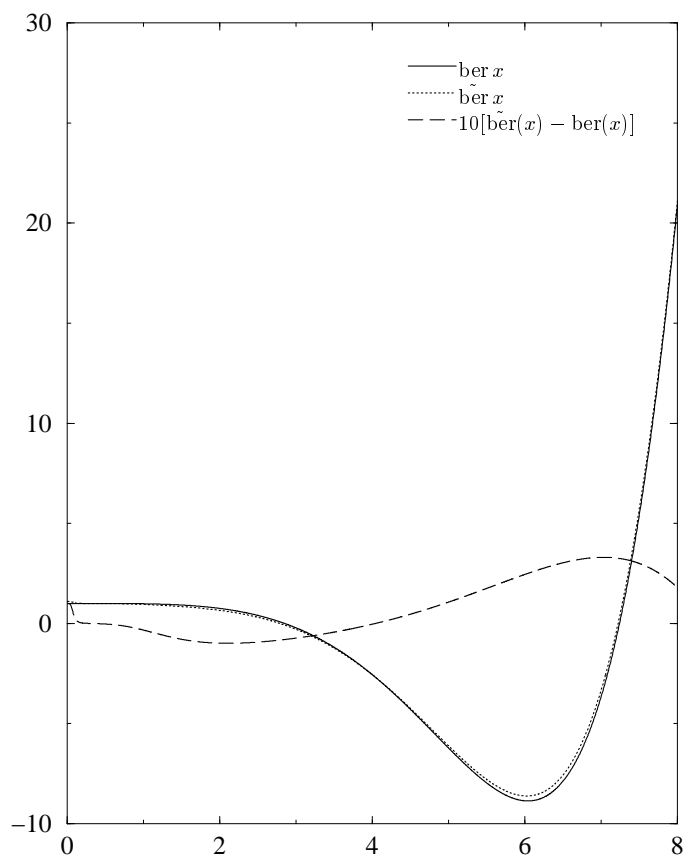

FIG. 1. Kelvin real function and its two-point quasifractional approximation, along with ten times the discrepancy between them.

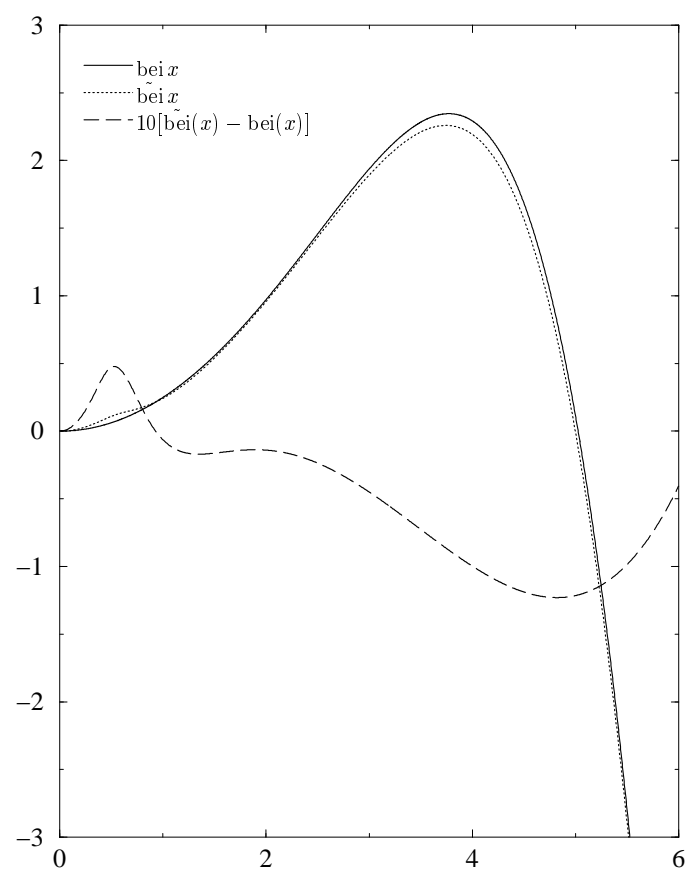

FIG. 2. Kelvin imaginary function and its two-point quasifractional approximation, along with ten times the discrepancy between them. 


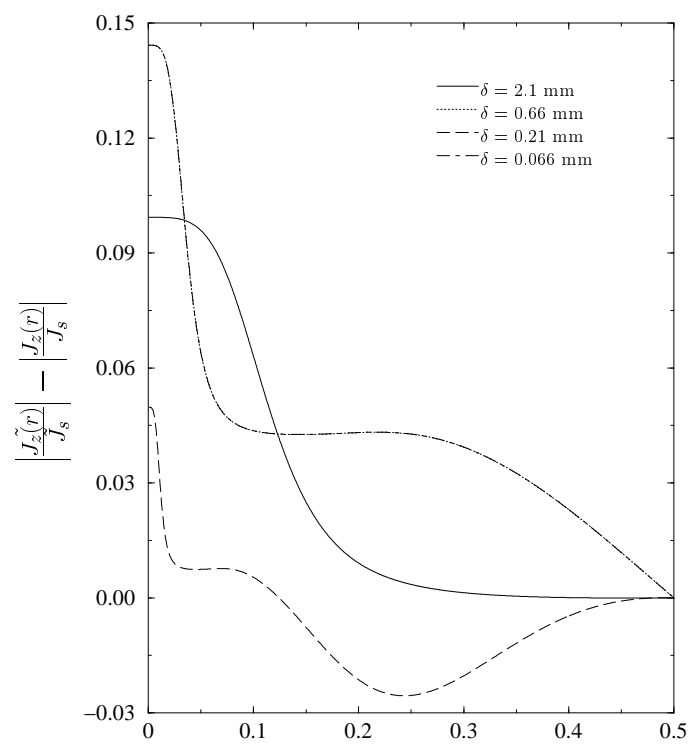

FIG. 3. Plots of the discrepancy between the analytical current distribution within a cylindrical conductor and the results obtained with two-point quasifractional approximation for four different values of the skin depth. 